\title{
«EL VALLENATO VALLENATO SE HABLA CANTAO’»: ALGUNAS ACTITUDES LINGÜÍSTICAS HACIA EL ESPAÑOL HABLADO EN VALLEDUPAR (COLOMBIA)
}

\author{
Aura Rosa Salazar Caro \\ Universidad Popular del Cesar (Colombia) \\ aurarosasalazar@unicesar.edu.co \\ Raúl Rodríguez López \\ Universidad Popular del Cesar (Colombia) \\ randresrodriguez@unicesar.edu.co
}

Recibido: 04/08/2020 - Aprobado: 14/09/2020 - Publicado: 15/04/2021

DOI: doi.org/10.17533/udea.lyl.n79a02

\begin{abstract}
Resumen: Este artículo analiza cómo definen los nativos de Valledupar su habla propia. En términos teóricos, este estudio acoge el modelo mentalista de tres componentes sociolingüísticos por medio de un estudio exploratorio con enfoque mixto que sigue el método directo. Los resultados revelan que los hablantes consideran que en Valledupar se habla cantao y, por otra parte, consideran que el español hablado en Valledupar es más prestigioso que el de otras variaciones de la región y, además, reconocen fenómenos de variación lingüística en la ciudad.

Palabras clave: Valledupar; vallenato; actitudes lingüísticas; español hablado en Valledupar.

\section{«EL VALLENATO VALLENATO SE HABLA CANTAO’»: SOME LINGUISTIC ATTITUDES TOWARDS THE SPANISH OF VALLEDUPAR (COLOMBIA)}

\begin{abstract}
This article analyzes how the natives of Valledupar define their own speech. In theoretical terms, this study embraces the sociolinguistic three-component mentalist model through an exploratory study with a mixed approach that follows the direct method. The results reveal that the speakers consider that Spanish is spoken cantao in Valledupar and, on the other hand, they consider that the Spanish spoken in Valledupar is more prestigious than other variants in the region and, in addition, they recognize some linguistic variations in the city.
\end{abstract}

Key words: Valledupar; vallenato; linguistic attitudes; Spanish of Valledupar. 


\section{Introducción}

$\mathrm{L}$

os estudios de actitudes lingüísticas a nivel intradialectal facilitan la comprensión de fenómenos como el prestigio lingüístico, la variación diastrática e incluso diatópica y el estatus que los hablantes le atribuyen a sus propias hablas locales. Blas Arroyo (1999, como se citó en Edwards, 1982) señaló que la investigación empírica sobre actitudes ha demostrado que los acentos regionales, de grupos étnicos minoritarios o de las clases bajas generan reacciones o juicos desfavorables en términos de estatus y prestigio.

Por su parte, Bernal (2016) analizó las actitudes de los bogotanos acerca de los dialectos del español de Colombia y concluyó que los hablantes tienen lealtad lingüística para con su superdialecto. Las actitudes positivas se orientan hacia la región andina; los informantes consideran que en Bogotá se habla el mejor español, que los costeños carecen de formalidad y cortesía, y que los regionalismos léxicos son inapropiados, en la medida en que no se corresponde con el habla de Bogotá.

Este artículo surge, pues, de un proyecto sobre las actitudes lingüísticas de los vallenatos ${ }^{1}$ hacia el español hablado en Valledupar. En este estudio se analizan las principales creencias de los nativos de Valledupar acerca de su variedad de habla, a partir de aspectos relacionados con la caracterización y el reconocimiento de rasgos lingüísticos propios y de fenómenos de variación a nivel diatópico y diastrático. Se acogen el muestreo propuesto por el Proyecto para el Estudio del Español de España y América (PRESEEA) y algunos apuntes sobre representatividad de las muestras planteados por Silva-Corvalán y Arias (2017).

Este texto se estructura en cinco partes. En la primera se ofrece una contextualización de Valledupar en términos geográficos y lingüísticos. En lo que concierne a la investigación, la segunda incluye la metodología que sustenta el trabajo. Luego, la tercera sección contiene el análisis de los datos, y en la última, las conclusiones. El análisis se hizo desde el modelo mentalista multicomponencial², específicamente del componente cognitivo que incluye los estereotipos sociales, las creencias y las percepciones que construyen los sujetos (López Morales, 1989, como se citó en Blas Arroyo, 1999) y que se comparten en una comunidad de habla. Por eso se incluyen en este artículo aspectos como la caracterización de la variedad lingüística propia y el reconocimiento de fenómenos de variación al interior de Valledupar.

\footnotetext{
1. $\quad$ En este artículo se usa vallenato generalmente como gentilicio de Valledupar. Se decidió emplear este término en lugar de valduparense, debido a que es el más usado en la ciudad y con el que más se identifican los nativos, como lo muestra el estudio de Estrada y Pérez (2019). En algunas ocasiones se hace referencia al vallenato como género musical o como variedad lingüística propia de la ciudad, pero en cada caso se hace la aclaración respectiva en una nota al pie de la página.

2. Este es un modelo que ha que ha sido discutido desde la sociolingüística Entre quienes apoyan su uso se encuentran Rojo (1979), Gómez Molina (2002), Drevdal (2009), González (2009) y Makuc (2011).
} 


\section{Contexto geográfico y lingüístico de Valledupar}

Valledupar es la capital del departamento del Cesar (Colombia), ciudad situada en el valle que forman los ríos Cesar y Guatapurí, y entre la Sierra Nevada de Santa Marta y la Serranía del Perijá. Tiene una extensión de 4493 $\mathrm{km}^{2}$ (ver Mapa 1), rodeados de montañas y atravesados por el río Guatapurí (Fundación Festival de la Leyenda Vallenata, 2014).

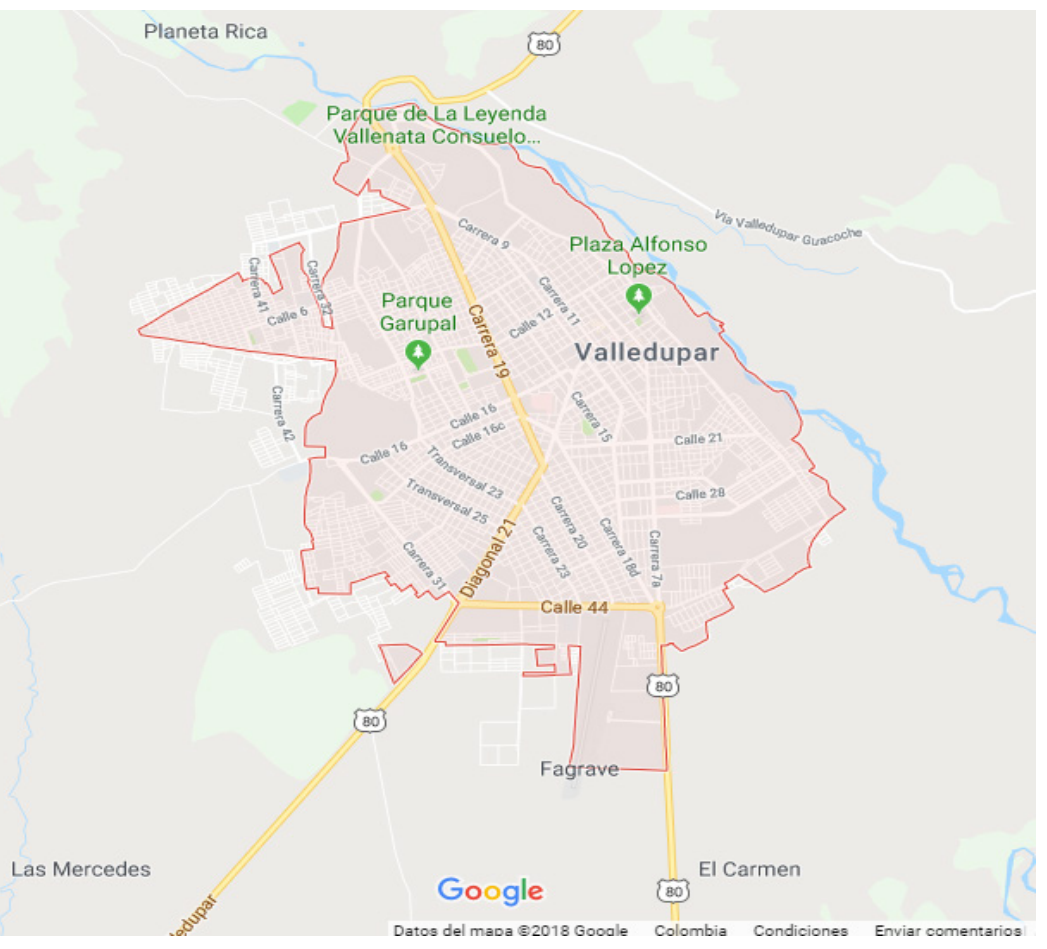

Mapa 1.Delimitación de Valledupar. Fuente: https://www.google.com.co/maps/@10.4671319,-73.2721939,13z

Es conocida como la «Ciudad de los Santos Reyes del Valle de Upar» y la «Capital mundial del vallenato» ${ }^{3}$. En Valledupar se llevan a cabo distintas festividades, el más importante es el Festival de la Leyenda Vallenata, cuyos antecedentes están vinculados con las llamadas fiestas del Milagro de la Virgen del Rosario. A partir de 1968, a la fiesta religiosa se le adicionó la música de acordeón como componente cultural y regional, para darle forma a uno de los festivales populares más importantes y concurridos de Colombia y del mundo (Martínez, 2017).

El municipio de Valledupar tiene una proyección para el 2020 de 532.956 personas, de las cuales se estima que 468.165 estaán en la cabecera municipal y 64.791 habitan los territorios rurales (DANE, 2018). La cabecera municipal está formada por seis comunas y 175 barrios $^{4}$. Durante los últimos cinco años la ciudad ha experimentado

3. El término vallenato se refiere al género musical declarado patrimonio cultural e inmaterial de la humanidad por la Organización de las Naciones Unidas para la Educación, la Ciencia y la Cultura (Unesco).

4. Datos obtenidos del portal valledupar.com 


\section{Algunas actitudes lingüísticas hacia el español hablado en Valledupar (Colombia)}

un boom demográfico, debido a que el incremento poblacional superó la media nacional del $6 \%$, por lo que pasó de tener 78.175 hogares en 2005 a 127.587 en 2018 (Barrios, 2019).

En términos lingüísticos, hay que señalar que existen pocos estudios acerca del español hablado en Valledupar. La caracterización de esta variedad se hace desde lo que han planteado sobre el dialecto costeño autores como Montes Giraldo (2012), Trillos (2012) y Cury Lambraño (2006), así como a partir de las investigaciones de Calderón y Gutiérrez (2006), y Olmos, Gómez y Pérez (2010). En la clasificación dialectal del español de Colombia, Montes Giraldo (1982, 2012) plantea dos superdialectos: el continental interiorano y el costero insular. De este último, conocido como dialecto costeño, hacen parte, por el lado de la costa atlántica, los departamentos de Córdoba, Sucre, Bolívar, Atlántico, Magdalena, Cesar, y parte de Chocó y Antioquia.

A partir de dicha clasificación, Montes Giraldo (2012) ubica el departamento del Cesar en el superdialecto costeño, al que caracteriza por el tratamiento de /-s/, la neutralización de la vibrante simple alveolar sonora / $/$ y la lateral alveolar sonora /1/ implosivas (/pielna/, /calne/) y la frecuencia de la nasal velar/-y/ (/pay/, /may/). El mismo autor establece, además, que es necesario diferenciar el dialecto costeño del atlántico —en el que se impuso el tuteo como trato general de confianza - del dialecto costeño pacífico, caracterizado por el voseo. Aclara, más adelante, que en algunas zonas del departamento del Cesar y en San Basilio de Palenque predomina el voseo. En concreto, el investigador define que en el dialecto costeño atlántico es frecuente la asimilación de la vibrante simple alveolar sonora /r/ y la lateral alveolar sonora /l/ implosivas a la consonante siguiente (/pacco/, / modde/, /puetta/, /cagga/). Por otra parte, Trillos (2012) plantea que el habla vallenata con dispersión hacia el sur de La Guajira se caracteriza por un escaso debilitamiento de /s/ implosiva y por la realización plena de la vibrante simple alveolar sonora /r/ y la lateral alveolar sonora /1/ en posición implosiva ([tarde], [balde], [altura]). Según esto, Valledupar se aleja de esa norma de asimilación de consonantes implosivas establecida por Montes Giraldo (2012) para el dialecto costeño atlántico, pero mantiene rasgos como la elisión de la vibrante simple alveolar sonora / $\mathrm{f}$ / en final de palabra (Terraza, 2020) y la frecuente aspiración de /s/ al final de sílaba interna y al final de palabra (Gómez, Olmos \& Pérez, 2010).

En el plano de los estudios de Valledupar se encuentra el trabajo «Caracterización sociolingüística de la comunidad de habla de Valledupar», de Calderón y Gutiérrez (2006). El objetivo de esta investigación fue determinar las actitudes y creencias de los nativos de Valledupar. De los resultados se puede resaltar lo siguiente: primero, que los hablantes afirman su sentido de pertenencia a su comunidad y definen a los nativos como personas dignas de confianza y aptos para ocupar cargos prestigiosos. Segundo, que, aunque a nivel regional los informantes consideran que el habla de Valledupar es más prestigiosa que el de otras ciudades capitales del Caribe, a nivel nacional reconocen como más prestigiosas las hablas de ciudades como Medellín, Cali, Bogotá o Bucaramanga y ubican a Valledupar en un punto intermedio.

La investigación anterior es el principal referente que se tiene sobre el estudio de las actitudes en Valledupar; sin embargo, es necesario señalar varias limitaciones metodológicas del estudio en cuestión: la primera limitación es que no se evidencia una postura teórica y metodológica clara con respecto a las actitudes. La segunda es que 


\section{Algunas actitudes lingüísticas hacia el español hablado en Valledupar (Colombia)}

en en el análisis solo se presentan datos cuantitativos. No obstante, hay que reconocer el valor de un ejercicio investigativo como este en una comunidad donde hay pocos estudios al respecto; además, hay que tener en cuenta que la información incluida en este artículo corresponde, según los autores, al primer informe de investigación. Por tanto, el presente estudio pretende ampliar los antecedentes y rescatar el valor de los datos cualitativos en los estudios de actitudes lingüísticas.

Entretanto, Gómez, Olmos y Pérez (2010) desarrollaron el estudio «La variación sociolingüística de /s/ implosiva en la comunidad de habla de Valledupar» para explicar la variación de /s/ al final de sílaba interna y al final de palabra. Esta investigación se hizo a partir del corpus PRESEEA. De las 54 entrevistas, se tomaron 18 y se analizaron con el programa SPSS. La observación se hizo mediante análisis perceptivo y confirmación espectrográfica mediante una muestra aleatoria de la selección léxica con la ayuda del software PRAAT. Se analizaron tres variantes del fonema /s/ en coda final: la retención de la sibilante [s], la aspiración [h] y la elisión (Ø). Con relación a las variables extralingüísticas independientes se analizaron las tres consideradas para el PRESEEA: grupo generacional, sexo y nivel de instrucción.

Para Valledupar se ratifica la presencia mayoritaria de la aspiración con un 51,9 \%, pero aparece la elisión del fonema /s/, con un 26,3\%, y la realización plena, con un $21 \%$. La elisión y la realización plena tienen más altos porcentajes en los hombres (57,1 \% de elisión y 55,0 \% de realización), que en las mujeres (42,9 \% de elisión y 45,0 \% de realización plena). Las mujeres, en cambio, utilizan más aspiraciones que los hombres. En cuanto a la variable generación, el fonema /s/ se realiza pleno por la generación 3 porque es el grupo más cercano a la conservación de la variante de prestigio. La generación 1 elide $(37,6 \%)$ y aspira mayoritariamente $(33,5 \%)$ en comparación con la realización plena del sonido (11,9\%). La generación 3, por su parte, aspira en un 27,2 \%, elide en 27,9 \% y pronuncia de forma plena en 45,3 \%. En cuanto al nivel de instrucción, resultó que el fonema /s/ se realiza pleno en el nivel de instrucción 3, mientras que en el nivel 1 se presenta un comportamiento lingüístico inverso al nivel de instrucción 3. A partir de estos resultados, se infirió que los niveles 2 y 3 conservan porcentajes muy cercanos en lo referido a la elisión y a la aspiración por lo que se diferencian únicamente en la realización plena.

Entre las conclusiones de este estudio, se definió, en primer lugar, que los hombres son más conservadores y las mujeres más innovadoras en la producción del fonema /s/. En segundo lugar, se detectó que entre las variables sociales estudiadas son más dominantes el nivel de instrucción y la generación en la variación del fonema /s/; y, por último, según los autores, se consideró que la investigación constituye un avance en la consolidación del estado del arte sobre los estudios sociolingüísticos en Valledupar.

En un estudio reciente, Terraza (2020) analizó la realización de la consonante vibrante simple alveolar sonora /r/ en posición final de verbos infinitivos en Valledupar, a partir de 36 entrevistas del corpus PRESEEA-Valledupar (2005). El autor observó la realización de las variantes [r] simple o plena (vibrante con oclusión), [I] aproximante (vibrante sin oclusión) y elidida ( $\varnothing$ cero fonético) de / / / en posición final de verbos infinitivos, teniendo en cuenta las variables lingüísticas categoría gramatical y posición del fonema /r/, y contexto fónico siguiente. De este 


\section{Algunas actitudes lingüísticas hacia el español hablado en Valledupar (Colombia)}

estudio, resulta que la variante de / / / en posición final de verbos infinitivos más frecuente es la elisión $(\varnothing)$, seguida por la aproximante [I], además de que las variables sociales que más inciden en la realización de la vibrante simple son la edad y el nivel de instrucción.

Con respecto a la edad, los hablantes de la primera generación (de 20 a 34 años) son los menos conservadores en el mantenimiento de la vibrante simple, en la tercera generación (de 55 años en adelante) hay mayor tendencia a la elisión. En la segunda generación (de 35 a 54 años) también se registra elisión de la vibrante simple, pero hay mayor tendencia a la realización aproximante. En cuanto a la variable nivel de instrucción, se comprobó que los informantes de niveles bajos son los que más eliden la vibrante simple, aunque la elisión esté generalizada en todos los niveles de instrucción de la comunidad de Valledupar.

\section{Cuestiones de método}

Este es un estudio con enfoque mixto ${ }^{5}$ en el que se reconoce la importancia de complementar datos cuantitativos con datos cualitativos para abordar un fenómeno psicosocial como el de las actitudes lingüísticas ${ }^{6}$. Los enfoques cualitativo y cuantitativo tienen limitaciones, por lo que emplearlos separadamente reduce las actitudes de los hablantes a cierto tipo de datos. Por ello, Salgado (2007, p. 1) planteó que «ambos enfoques utilizados conjuntamente pueden enriquecer el proceso de la investigación científica de manera importante, ya que no se excluyen ni se sustituyen, sino que se complementan». En esta misma línea, Casanova (2000) reconoció que la metodología de las ciencias sociales puede resultar muy útil en sociolingüística debido a que posibilita, gracias a la combinación de técnicas cualitativas y cuantitativas, la caracterización de una realidad determinada a partir de una muestra o segmento de ella.

Izquierdo (2003) señaló al respecto que «el estudio de las actitudes lingüísticas se ha planteado desde dos perspectivas distintas, si bien con resultados que pueden ser complementarios: la mentalista y la conductista» (p. 20). Este trabajo se enmarca en la perspectiva mentalista ${ }^{7}$ desde la cual se asume que las actitudes, por su naturaleza psíquica y su carga cognitiva, pueden ser inferidas a través de métodos introspectivos centrados en los discursos o las respuestas verbales de los sujetos ante determinadas cuestiones (Campos, 2018).

En términos concretos, esta investigación se inscribe en el método directo, en el cual se interroga directamente sobre las opiniones, juicios y valoraciones de los hablantes frente a una lengua. En el método indirecto, por su parte, se pretende llegar a conocer las actitudes por medio de grabaciones y cuestionarios de pares ocultos, por lo

5. Este enfoque se ha planteado desde diferentes autores como enfoque múltiple, diseño mixto, integrado, o multimodal (Bryman, 2006, como se citó en Blanco \& Pirela, 2015). Más allá de la denominación, es necesario asumirlo como una opción para investigar a partir del uso conjunto de diversos métodos y técnicas para el estudio de un mismo problema, considerando diferentes criterios que integren procedimientos para validar el conocimiento científico (Blanco \& Pirela, 2015).

6. Blanco y Pirela (2015) plantearon que quienes están a favor de una visión múltiple en investigación reconocen que «el uso conjunto de ambos enfoques logra optimizar y validar los resultados del proceso de investigación científica de forma significativa» (p. 104).

7. Esta es una perspectiva que ha generado mucha controversia. Sin embargo, Blas Arroyo (1999) y Campos (2018) reconocieron que es la que tiene mayor desarrollo y tradición científica, sobre todo en el campo de las actitudes lingüísticas. 


\section{Algunas actitudes lingüísticas hacia el español hablado en Valledupar (Colombia)}

que es común que el uso de técnicas como la matched-guise y la escala del diferencial semántico (Castillo, 2006). En este sentido, Erdösová (2011) afirmó que a los métodos directos pertenecen los cuestionarios de preguntas tanto abiertas como cerradas y las entrevistas.

En cuanto a la metodología de este estudio se refiere, se aplicó, en primer lugar, una entrevista semiestructurada, que incluía una grabación de audio que podría considerarse una variación de la técnica de pares ocultos. En este sentido, se logró integrar dos métodos: por un lado, se interrogó directamente a cada hablante sobre sus percepciones acerca de su lengua; y, por otro, se le hizo escuchar un audio en el que debía reconocer las características de su comunidad de habla y someterla a una valoración en términos de prestigio lingüístico ${ }^{8}$.

En los estudios de actitudes lingüísticas la entrevista semiestructurada es uno de los procedimientos más utilizados (González, 2008). En el caso de esta investigación, se diseñó un cuestionario mixto de 25 preguntas abiertas y cerradas. Al respecto, González (2009) señaló que las preguntas abiertas abren la posibilidad de acceder a las explicaciones que los informantes aducen en los puntos más candentes. Este tipo de preguntas permite organizar el análisis de los datos desde los discursos y las categorías dadas por los mismos hablantes. Por otro lado, las preguntas de respuesta cerrada permiten cuantificar, analizar la incidencia de las variables sociales y comparar los datos con los obtenidos en investigaciones similares (González, 2009).

\subsection{Criterios de selección de la muestra}

La selección de la muestra se hizo a partir de las directrices metodológicas del proyecto PRESEEA (2003). En este estudio la mayoría de informantes fueron todos nativos, salvo dos excepciones que cumplen con el criterio de haber llegado a la ciudad antes de los diez años, provenientes de otros municipios del departamento del Cesar ${ }^{9}$. Se entrevistó a 36 nativos de Valledupar ${ }^{10}$, seleccionados mediante un muestreo por cuotas con afijación uniforme, que consiste en «dividir el universo relativo en subpoblaciones, estratos o cuotas —atendiendo a las variables sociales determinadas - y en asignar igual número de informantes a cada una de esas cuotas» (PRESEEA, 2003). La muestra es representativa teniendo en cuenta la naturaleza del fenómeno a ser analizado y los recursos disponibles para los investigadores (Silva-Corvalán \& Arias, 2017). A partir de esto, las variables sociales propuestas por PRESEEA (2003) son las siguientes: sexo, grado de instrucción y edad. Cada una de ellas se organizó de la siguiente manera:

8. $\quad$ Por cuestiones de espacio, las preguntas asociadas al audio se analizarán en un próximo artículo.

9. $\quad$ En PRESEEA se plantea que los hablantes candidatos a informantes deben haber nacido en la comunidad, haber llegado a la misma antes de cumplir los diez años o llevar viviendo allí más de veinte años, y que, en caso de tratarse de un residente no nativo, su origen lingüístico no debe ser marcadamente diferente (PRESEEA, 2003).

10. Todos los hablantes que integran la muestra fueron informados sobre el proyecto y sus alcances, por lo que decidieron participar de manera voluntaria. De este modo, se respetó el principio de consentimiento informado, en el que «los informantes han de haber accedido voluntariamente a participar en la investigación y a saber lo que conlleva» (Hernández-Campoy 2005, p. 152). 


\begin{tabular}{|c|c|c|c|c|c|c|}
\hline \multirow{2}{*}{ VARIABLES } & \multicolumn{3}{|c|}{ Generación 1 } & \multicolumn{2}{c|}{ Generación 2 } & \multicolumn{2}{c|}{ Generación 3 } \\
\cline { 2 - 7 } & Hombres & Mujeres & Hombres & Mujeres & Hombres & Mujeres \\
\hline Grado de instrucción 1 & 2 & 2 & 2 & 2 & 2 & 2 \\
\hline Grado de instrucción 2 & 2 & 2 & 2 & 2 & 2 & 2 \\
\hline Grado de instrucción 3 & 2 & 2 & 2 & 2 & 2 & 2 \\
\hline
\end{tabular}

Tabla 1. Distribución de la muestra según las variables sociales del PRESEEA (2013).

\section{Análisis de los datos}

\subsection{En Valledupar se habla cantao'}

Una de las cuestiones fundamentales que se abordó concierne a las creencias de los nativos sobre el habla de Valledupar. La primera pregunta de este aspecto fue abierta y las opiniones fueron tan diversas que complejizaron una posible cuantificación de los resultados. Así, pues, algunos hablantes consideran que en Valledupar se habla cantao. En sus palabras:

con un cantadito / como un dejo en ciertas palabrah / una prolongación (CENTRO1532M) ${ }^{11}$.

eh una prolongación de cierta letra que suena a ser como cantaíto/(CENTRO1532M).

cantaíto / nosotros hablamoh eh cantao /veee / y tú donde estabah / tú qué estáis haciendo/ (LAGUAJ2013H).

es como cantado / oooye mira / veee / es así máh o menoh/ (VALLEMC0122M).

de a donde vení / pa' onde vai // que traei ahí// como te llamaí/ (PARAISO0813H).

Como se observa, algunos relacionan el cantao con el alargamiento de las palabras ${ }^{12}$. Otros lo ejemplifican desde el uso de la forma veee con la aliteración de la vocal semiabierta anterior, una expresión característica del habla de Valledupar variante del verbo ver conjugado en la segunda persona del singular del modo imperativo afirmativo (ve). De acuerdo con la clasificación de Rodríguez (1999), se podría decir que veee es un marcador discursivo apelativo. En Valledupar, esta forma verbal se usa con la intención de captar la atención del interlocutor (oye mira/ veee // veee/ y tú donde estabah) ${ }^{13}$, o para indicar sorpresa o asombro ante un hecho inesperado (veeeee

11. Lo que aparece entre paréntesis corresponde al código que le fue asignado a cada informante a partir del orden de realización de cada entrevista. En este caso, CENTRO1532M corresponde al informante número quince. El código presenta la siguiente información: CENTRO, barrio Centro. 15: número del informante. 3: grado de instrucción, estudios profesionales. 2: segunda generación, 35-55 años. M: sexo, mujer. Los mismos detalles se especifican para cada informante.

12. Este fenómeno podría asociarse con lo que plantea Cury (2004) sobre la formación del verso antiguo en el que las silabas largas y las breves se alternan acompasadamente para producir melodía.

13. Algunos de estos discursos que aparecen como ejemplos son tomados directamente de las entrevistas, mientras que otros se obtuvieron en conversaciones con hablantes nativos que, aunque no hicieron parte de la muestra de este estudio, decidieron aportar para la explicación del fenómeno. 
Algunas actitudes lingüísticas hacia el español hablado en Valledupar (Colombia)

/ muchacha / todo eso es tuyo // veeee y quién compró ese pocotón de comía) ${ }^{14}$. Para los nativos, hablar cantao se relaciona también con el uso de formas verbales voseantes que pueden o no están precedidas por el pronombre de segunda persona del singular tú (tú qué estáis haciendo, pa onde vai / qué traei ahí // como te llamaí).

En algunos lugares de México también se suele considerar que se habla cantado. En ese sentido, Martínez (2017) señaló que mientras hacia el norte de México alargan la última sílaba hacia arriba; en regiones del sur les ponen más énfasis a los acentos de cada palabra ${ }^{15}$. Por otro lado, Serrano (2017) sostuvo que lo cantadito del habla de Guadalajara se debe «a una tendencia en subir la curva prosódica detrás de cada enunciado o segmento del habla» (p. 1). Según el autor, el hablar cantado suele asociarse con fenómenos prosódicos como el alargamiento en la pronunciación de las palabras. De acuerdo con lo anterior, podría decirse que hablar cantao está relacionado con la prolongación de elementos como la duración y el tono de los sonidos del habla. Es un rasgo suprasegmental de las variedades lingüísticas con características prosódicas semejantes a las de los sonidos musicales, por lo que es posible comparar la acción de hablar con la de interpretar una melodía.

Otros hablantes consideran que en Valledupar se habla golpiado. Este hecho plantea también una discusión en torno a lo que significa hablar golpiado, una expresión sobre la que poco se ha dicho en términos teóricos. Frente a esto, los nativos consideran que:

hablamos golpiado pero no tan golpiao como otrah regiones (GARUP2131M).

Yo diría que muy relajado / aquí el ehpañol eh muy relajao / se utilizan términoh relajado y tajanteh / (CANDN3322H).

La mayor parte de lah palabrah / ajá / a pue / va pue // lah golpean / el acento de lah última palabrah lah omiten (LUISCG2331M).

En Montería, Salazar (2019) identificó que, para los nativos, hablar golpeado comprende varios fenómenos de los señalados por Flórez (1949) como propios del habla monteriana y algunos de los rasgos que Montes (1996, 2012) identificó como característicos del superdialecto costeño. De acuerdo con lo anterior, el término goppiao ${ }^{16}$ se relaciona con la presencia de fenómenos como la elisión de /d/ intervocálica, la pérdida de /s/ en posición implosiva y la elisión de / $/$ al final de palabra. En ese sentido, aunque el habla de Valledupar comparte los principales rasgos lingüísticos del Caribe, se diferencia en algunos aspectos de otras zonas, lo que probablemente explique por qué un informante señala que se habla golpiado ${ }^{17}$ pero no tan golpiao como en otras regiones. Otro informante relaciona esta particularidad con la elisión de sonidos al final de las palabras y las consecuentes

14. Algunas veces se usa para indicar incredulidad frente a lo que el interlocutor expresa. Como puede verse, puede aparecer como de apertura o como de cierre, dependiendo del contexto y la intención comunicativa.

15. Según el mismo autor, también se suele decir que en este territorio hablan «aporreado» Este término podría considerarse una variante de la expresión hablar goppiao,que se analizará más adelante.

16. Se emplea el término goppiao porque así dicen comúnmente los hablantes de Córdoba. En esa sola expresión se dan varios fenómenos fonéticos que conviene analizar en este artículo. Tomando como base la forma gol-pe-a-do, se observa que en la última sílaba se elide la aproximante dental al inicio. En las penúltima y antepenúltima sílaba hay un hiato «e-a» que se diptonga en el grupo -ia-, y en la trasantepenúltima sílaba se asimila /1/ a la consonante siguiente, que en este caso es /p/. Se podría decir que esta es una expresión que explica en sí misma lo que significa.

17. En Valledupar, algunos hablantes dicen golpiado, otros golpiao pero nunca goppiao. Hay que recordar que en esta región no se produce el fenómeno de asimilación de /1/ implosiva a la consonante siguiente, que es muy característico de Montería. En este artículo se emplean cada una de las variantes del término, dependiendo del contexto al que se aluda. 


\section{Algunas actitudes lingüísticas hacia el español hablado en Valledupar (Colombia)}

implicaciones prosódicas que genera ese fenómeno, por lo que considera que en Valledupar se «golpean» las palabras.

A partir de lo anterior, hay quienes consideran que en Valledupar se habla «normal». Otros dicen que se habla «con influencia guajira», o «diferente a como se hablaba antes». Hay un grupo que, por otra parte, reconoce algunas diferencias entre el acento actual del español y el de hace cuarenta años. En este grupo se encuentran principalmente personas de la tercera generación, quienes argumentaron que:

El hablar del valduparense hace cincuenta años era diferente al de ahora (12DEOCT1633).

Lo que pasa es que el idioma típico antiguo del vallenato se ha ido acabando (12DEOCT0633H).

Como puede notarse, las personas de la tercera generación ${ }^{18}$ reconocen que la generación actual ha adoptado formas de hablar distintas a lo que ellos denominan el vallenato vallenato ${ }^{19}$. Con respecto a cómo se habla en Valledupar, se podría resumir que los vallenatos se debaten entre hablar cantao, golpiado, «relajado», «normal», «con influencia guajira»y «diferente a como se hablaba hace unas décadas».

En las respuestas a la pregunta anterior, algunos nativos consideran que en Valledupar se habla golpiado. Sin embargo, los datos cuantitativos revelan que la mayoría no comparte esta creencia. En el Gráfico 1 se observa que el $84 \%$ de los participantes considera que en Valledupar no se habla golpiado. Incluso, una parte de los hablantes argumentó que quienes hablan golpiado son las personas de los departamentos de Sucre, Bolívar y Córdoba. Al respecto, cabe añadir que el habla en estos tres departamentos suele asociarse por compartir muchos rasgos lingüísticos, probablemente por su cercanía geográfica. Es necesario recordar en este punto que hasta principios del siglo xx Córdoba y Sucre formaban parte de la jurisdicción del hoy departamento de Bolívar. De hecho, en su estudio sobre el español de Montería y Sincelejo, Flórez (1949) denominó a estos territorios como «poblaciones bolivarenses», e incluye esta variedad lingüística en lo que denomina «el habla bolivarense» (p. 126).

\footnotetext{
18. Las otras variables sociales no fueron tenidas en cuenta debido a que no incidieron de forma significativa en los resultados de las temáticas que se analizan en este artículo.

19. Vallenato vallenato es la calificación que dan los hablantes a su variedad lingüística originaria.
} 


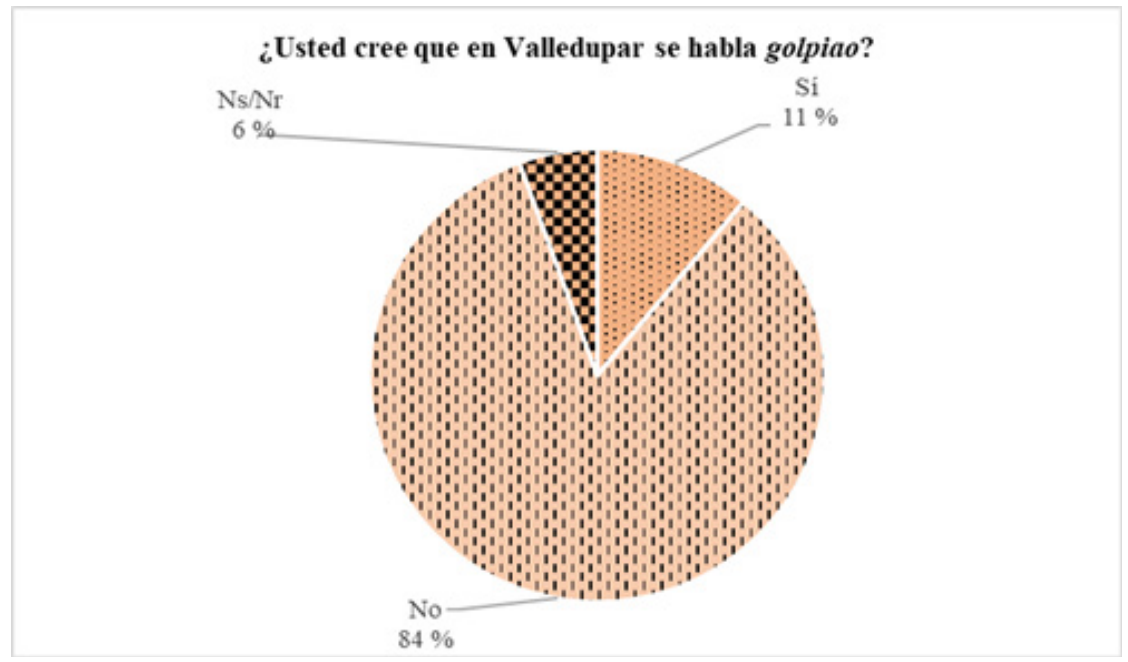

Gráfico 1. ¿Usted cree que en Valledupar se habla golpiao?

En términos generales, cabe precisar qul español hablado en Valledupar comparte con el resto de la región caribe colombiana rasgos lingüísticos como la aspiración o pérdida de /-s/implosiva, la elisión de /d/ intervocálica, la elisión de /r/ al final de palabra y el uso de la forma apocopada pa en lugar de para. Sin embargo, no presenta asimilación de / $\mathrm{f} /$ a la consonante siguiente en la producción de vocablos como parque /'pakke/, porque /'pokke/ o puerta /'pwetta/. Aunque se suele considerar que el hablar "golpeado» se caracteriza por la aspiración de /-s/ implosiva (Betancourt \& García, 1998), en realidad se trata de un rasgo más complejo que abarca diversos fenómenos fonéticos característicos del superdialecto costeño. La mayoría de los vallenatos cree, pues, que no habla golpiado, porque suele relacionar este fenómeno con la asimilación de / / / a la consonante siguiente y este rasgo no es característico de su variedad lingüística.

Este componente se cerró con una pregunta relacionada con lo distintivo del habla de Valledupar. Las opiniones están también divididas, ya que en general los hablantes se refieren a aspectos diversos asociados a criterios de corrección o de prestigio (que es bien expresado // que no son tan vulgaren como en Barranquilla/), el uso de formas léxicas (ajá / ujum /el compa / el manito), verbales (vení ve / ve y tu onde ehtabah / vee /), o el empleo de formas discursivas características (los vallenatoh leh gusta por naturaleza apropiarse de lo narrativo / más allá de la forma de hablar eh la forma en que lo dice).

Algunos hablantes se alejan de los aspectos lingüísticos y orientan la respuesta hacia la forma de ser o el espíritu del vallenato (somos divertidos / alegreh / fiehteroh/; el cariño / el amor con que uno trata a lah personah de afuera/). Sin embargo, la mayoría de los informantes considera que lo distintivo del español de Valledupar es:

Loh acentoh / la forma que nosotroh le quitamoh letrah a lah palabrah/ (PARAISO0921H).

nosotroh hablamoh cantao pero no eh un cantado que se oye como / exagerado sino un cantadito lindo (COMFAC1422M).

Hablamos cantao y somos neutros (12DEOCT2921H).

Por el particular acento / estamos muy conectados con lo que es San Juan y Villanueva (VALLEM0331H).

El hablar cantao (PARAISO1011H; CAÑAGU1223H; LAGUAJ2013H; 12DEOCT2921H; CANDN3322H). 
Algunas actitudes lingüísticas hacia el español hablado en Valledupar (Colombia)

Como puede notarse, la característica más común señalada por los informantes es el hablar cantao. De ello, se puede deducir que en Valledupar se habla cantao, lo que significa que los hablantes reconocen que su variedad lingüística tiene rasgos prosódicos melodiosos que la distinguen de otras variedades lingüísticas del caribe colombiano y del país.

\subsection{Actitudes hacia la variación diatópica y diastrática en el español hablado en Valledupar}

En este componente de la investigación se asume la variación diatópica en términos del reconocimiento y la valoración de las diferencias existentes entre el habla de la capital del Cesar y las hablas de los municipios o pueblos circundantes a esta. Por ello, se preguntó a los informantes si consideran que en Valledupar se habla igual que en los pueblos o municipios cercanos.

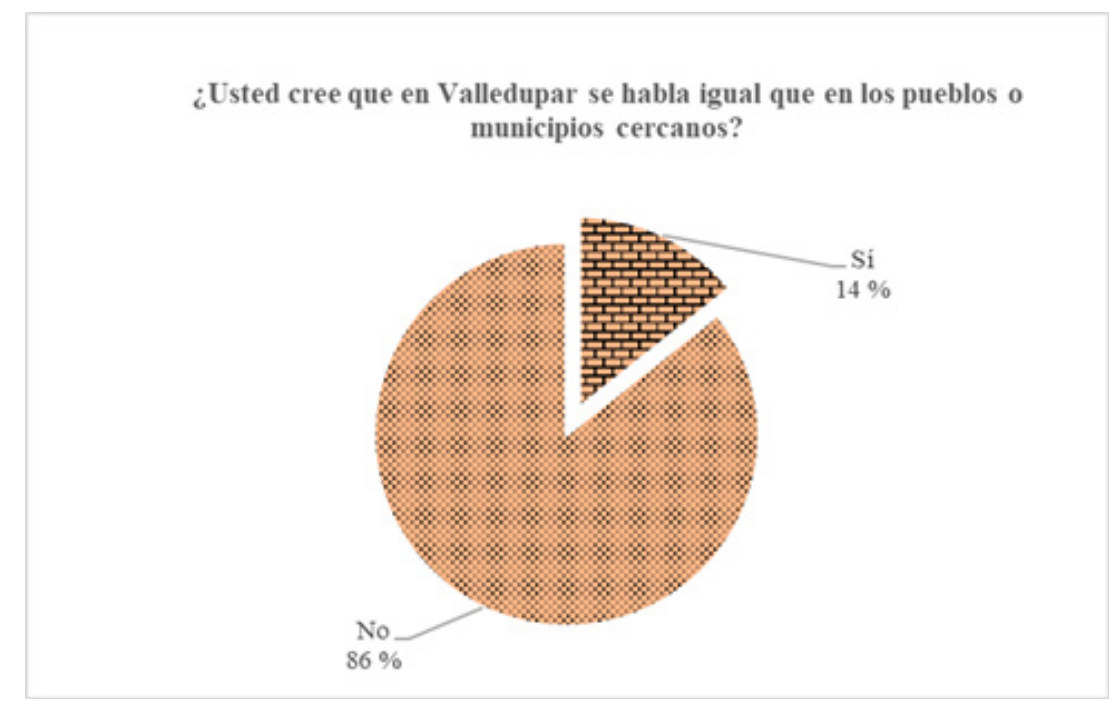

Gráfico 2. ¿Usted cree que en Valledupar se habla igual que en los pueblos o municipios cercanos?

Como se observa en el Gráfico 2, el $86 \%$ de los informantes coincide en que en Valledupar se habla diferente a como se habla en los pueblos o municipios cercanos. Hay hablantes que reconocen que, en los sectores rurales, dada la influencia de las lenguas indígenas, existen variantes del español que se habla en Valledupar. Algunos reconocen que entre más lejanos estén los pueblos, se tiende a hablar más cantao. En sus discursos señalan que:

[...] pero en San Diego lah ehpresioneh son totalmente diferentes / el cantaito es mucho máh alto/ (VALLEM0331H). No/señor / porque ellos hablan.../ por decir/ si comenzamos por los pueblitos estos que están cercanos / nunca hablan igual / siempre meten la pata como dicen / las cosas a lo derecho / siempre dicen las cosas al revés/ (KENN0713M). [...] tienen un dialecto diferente / por lo menoh allá en Guacoche [los imita gritando] ¡Veee y pa onde vaiii tú/ veee! ¿De onde veniii / tú ?/ veee! Y en el Alto de la Vuelta / Badillo / también hablan así / yo he tratao / tengo familia por ahí / así hablan elloh /gritao/ (PARAISO0813H).

[...] a medida que uno se va saliendo de Valledupar / se habla como máh cantaito / porque si tu vah a loh puebloh lo que eh Atanqueh / y como tienen máh cosah de loh indigenah entonceh tiene ese / que uno dice / así no se habla $\mathrm{o} /$ elloh no relacionan lah cosah igualeh/ (COMFAC1422M). 


\section{Algunas actitudes lingüísticas hacia el español hablado en Valledupar (Colombia)}

[...] el de los pueblos eh máh tímido / máh recogido / menos ehpontáneo / eh máh campesino /o sea eh máh montuno diría yo en ese sentido / eh una ehpresión que usa uno acá porque eh que el campesino /yo hago la diferencia entre un campesino y el de un pueblo mediano / Valledupar eh un pueblo mediano/ (ARIZ1833M).

Dihtinto/lo que eh en Atanque / lo que eh en.../ en.../ aquí en estoh pueblitoh que están aquí / que son corregimientoh / eso eh terrible / y si eh aquí en la Pah también / yo digo que en La Pah habla más / más / o sea no hablan un cohteño así / sino que tiene como un acento mah / mah / como mah pesaito/ (VILLAR3023H).

Los nativos de Valledupar reconocen las hablas rurales como diferentes a nivel fonético, prosódico, léxico, sintáctico, por lo que consideran que en los pueblos se habla como en La Guajira o más cantao que en la ciudad. En estos discursos, los espacios rurales se configuran mediante contenidos simbólicos asociados al atraso y a la falta de formación académica (siempre meten la pata, dicen las cosas al revés, eso es terrible). Algunos califican las variedades lingüísticas que se hablan en los pueblos como «antiguas», «raizales», «indígenas», «pesadas», «campesinas», «montunas» ${ }^{20}$. Todas estas son valoraciones negativas que sitúan a las hablas rurales por debajo de las urbanas y configuran imaginarios en torno a la ruralidad desde una perspectiva marginal, sustentada en la creencia de que las ciudades son los escenarios de desarrollo de las formas legitimas de hablar una lengua. Al respecto, Céspedes (2017) sostuvo que:

[...] el espacio pasa de ser considerado una categoría física y objetiva a ser una categoría cognoscitiva y subjetiva, lo que se manifiesta en las conceptualizaciones de los hablantes, las que algunas veces son estereotípicas de los pobladores en relación con valores negativos (p. 1).

De hecho, se puede establecer un contraste entre la manera como configuran los informantes las hablas rurales y urbanas. Los espacios urbanos se constituyen en símbolo de modernidad y de civilización, como se evidencia en los siguientes comentarios:

[...] tú vah a loh pueblitoh que nos rodean por aquí por ejemplo Guacoche / la Jagua / Aguah Blancah / San Diego / La Pah / hay mah gente hablando así el idioma raizal / ese que aquí en la ciudá / ya la ciudá se ha ido / modernizando / mucho en ese aspecto/ (12DEOCT0633H).

El dialecto costeño se habla muy bien es en la capital [...] / Sí / porque loh puebloh hablan muy diferente / ufff // mire / uno va aquí a Patillal / y elloh tienen un.../ tienen un estilo máh que todo de la Guajira / y eso es Cesar// [...] máh pueblo / pueblo pueblo / lah palabrah son más / como más rebuscadas / como máh antiguah/ (VALLEMC0122M). [...] no somos igual y ahí si veo que nosotroh somoh un poco yendo a la palabrah / un poco máh refinadoh / nosotroh hablamoh golpiado / pero no tan golpiao como los de los municipoh / allá si vah a encontrar personah que te hablan a ti máh golpeado y atravesado/ (GARUP2131M).

En estos discursos siguen latentes los juicios discriminatorios que atribuyen un mayor prestigio al habla urbana. En este caso, los vallenatos consideran que su acento es más refinado en comparación al de los habitantes de los pueblos cercanos. Sin embargo, se debe aclarar que no existe ninguna lengua, variedad, dialecto o acento mejor o superior que otro, pues cada lengua se inscribe en un contexto social y cultural que resulta adecuada para las necesidades comunicativas de sus hablantes (Trudgill, 1983). Por tanto, las valoraciones acerca de cómo se debe hablar se sustentan en el prestigio social del que gozan algunas variedades, concepto que a su vez está ligado a factores como la clase social, el poder económico y político y, por supuesto, la corrección gramatical (González Cruz, 1995; Hernández-Campoy, s. f.).

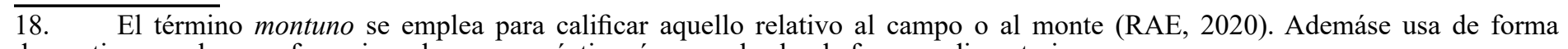
despectiva para hacer referencia a algo que es rústico, áspero o hecho de forma rudimentaria. 


\section{Algunas actitudes lingüísticas hacia el español hablado en Valledupar (Colombia)}

Por otro lado, en lo que respecta a la variación diastrática, se indagó sobre las creencias de los informantes de las diferencias entre la manera de hablar en diferentes sectores barriales y estratos socioeconómicos.

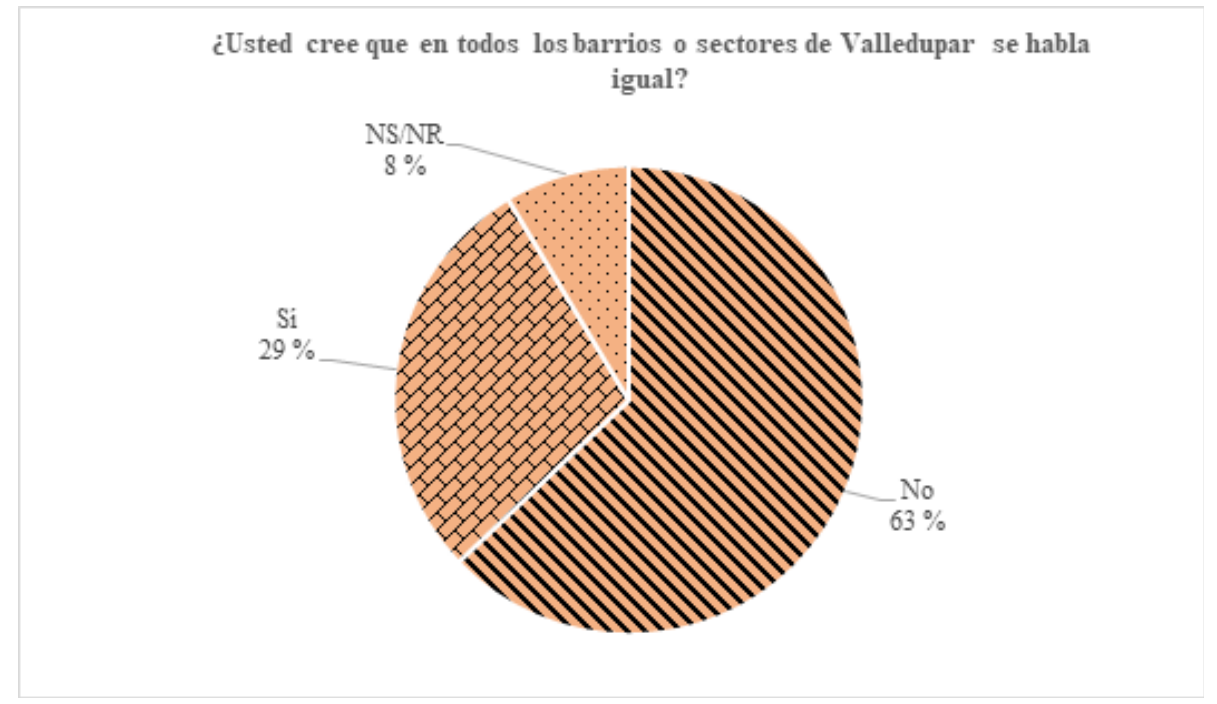

Gráfico 3. ¿Usted cree que en todos los barrios o sectores de Valledupar se habla igual?

En el gráfico anterior se muestra que el $63 \%$ de los informantes reconoce la variación sociolingüística y establece diferencias entre la manera de hablar de los sectores de estratificación social alta y la de los sectores de estratificación más baja. Con respecto a esto, los hablantes sostuvieron que:

La clase baja habla máh golpiao (GARUP2131M).

La clase baja tiende máh a estar hablando de una manera con máh acento máh golpiado / comiéndose lah palabrah/ (GARUP2131M).

Hay diferenciah /en otros barrios donde son / barrios más vulnerables / pienso yo que ahí sí eh verdá que se habla mucho peor/ (ÁLAMOS1131M).

Si tú te meteh a Novalito / allá lah niñah no van a hablá como te hablan por acá / sino todo delicado/(PARAISO1011H).

Tú sabes que loh del norte es pura gente high light / elloh tienen plata / hay unoh que lo miran al pobre por encima del hombro/ (VALLEM0212M).

Loh de la clase alta claro ehtá / si porque tu sabeh que no hay que negarlo / el ehtrato socioeconómico hace que se relacione con personah diferenteh ya / entonceh son máh ehtudiadoh / son máh reservadoh al momento de usar malah palabras / (PARAISO0921H).

Si va y por allá por la Villa Fuenteh tu encuentra a la gente hablando en un tono / too mundo habla gritado / hablan gritando y elloh vienen.../ esa eh una invasión y esa invasión tiene mucho arraigo de loh negro/ (1MAYO1332H).

La discriminación que se evidenció a nivel diatópico permanece también a nivel diastrático; es decir, en Valledupar se ve discriminación hacia las variedades de las clases bajas. Los vallenatos asocian las clases bajas con rasgos poco prestigiosos, como hablar golpiado, hablar «mucho peor» o hablar gritao; y las clases altas se definen como «más estudiados», «más reservados». A partir de ello, se detecta que los hablantes del norte de la ciudad valoran negativamente el habla de los sectores populares, teniendo en cuenta que hablar golpeado es lo opuesto a hablar refinado. Sobre esto, González Cruz (1995) consideró que:

[...] no hay nada inherente a las variedades de una lengua que les haga ser consideradas inferiores, se trata más bien 


\section{Algunas actitudes lingüísticas hacia el español hablado en Valledupar (Colombia)}

de las actitudes hacia sus hablantes, a quienes se cataloga como pertenecientes a un determinado grupo de escalafón bajo o no prestigioso (p. 717).

En estos discursos se nota una discriminación social y lingüística hacia las clases bajas, reconocida incluso por quienes pertenecen a estas clases no prestigiosas. Un hablante de un sector de alto riego, de estrato socioeconómico uno, valora positivamente a los hablantes de un barrio reconocido, puesto que define su modo de hablar como «delicado», atribuyéndole valores como la «finura» y la «sutileza». Este proceso es definido por Muñoz Navarrete (s. f) como automachaque, que está relacionado con la autoexclusión de las clases bajas al considerarse inferiores a nivel lingüístico y social que otros sectores sociales de mayor posición.

Sin embargo, es preciso señalar que esta discriminación trasciende los límites de lo sociolingüístico. El término invasión que aparece en los discursos implica la ocupación ilegal de un territorio ${ }^{21}$. Las invasiones son asentamientos socialmente vulnerables al margen de las ciudades que se encuentran en zonas de alto riesgo y sin acceso a los servicios públicos (El Espectador, 2000), considerándose negativamente por los sectores barriales externos a ellas. En los relatos analizados se las asocia con «raíces afrodescendientes» y con hablar gritao. Esta relación implica una discriminación lingüística y social porque se vincula a las personas pertenecientes a la comunidad negra con rasgos lingüísticos «indecorosos». A este respecto, Islas (2005) apuntó que «la discriminación se patentiza principalmente en las prácticas de exclusión y estigmatización, y que estas prácticas producen una desigualdad social y material, antes que una marginación lingüística» (p. 13).

Se puede ver entonces que, así como desde la ciudad se margina lo rural, al interior de la ciudad se estereotipan aquellos espacios físicos que son considerados marginales, y esta marginación se extiende a las personas de las comunidades negras, quienes son estigmatizadas a partir de la atribución de rasgos lingüísticos poco prestigiosos. Estos datos confirman el planteamiento de Edwards (1982, como se citó en Blas Arroyo, 1999), según el cual los estudios de actitudes demuestran que los acentos regionales o rurales, de grupos étnicos minoritarios o de las clases bajas evocan reacciones desfavorables en términos de estatus y prestigio lingüístico.

\section{A modo de conclusión}

A partir de los datos analizados, se puede concluir que para los hablantes una de las características del español hablado en Valledupar es que se habla cantao, es decir, hay en cada expresión un tono musical. Esto último resulta más interesante si se recuerda que la ciudad es considerada la capital mundial de la música vallenata y uno de los escenarios musicales y culturales de mayor reconocimiento en el país y en el mundo. Hay que señalar que esa musicalidad ha ido perdiendo fuerza en las últimas generaciones, como lo señalaron algunos nativos de la tercera generación (mayores de 55 años) y como puede notar cualquiera que visite la ciudad.

Segundo, hay entre los vallenatos un reconocimiento de la variación lingüística en términos diatópicos y diastráticos. Se cree que el español hablado en la capital del Cesar es distinto al que se habla en los corregimientos

19. En ese sentido, Ocampo (2003) la definió como asentamientos resultantes del proceso de ocupación de tierras. La autora las asocia como una de las actividades de los movimientos sociales en Córdoba (Colombia). 
o en los municipios cercanos. Esta diferenciación está determinada por imaginarios según los cuales en la cabecera municipal se habla mejor que en los territorios rurales y que en los barrios de estrato alto se tienen usos lingüísticos mucho más prestigiosos que en los sectores considerados marginales. En definitiva, los nativos de Valledupar le atribuyen mayor prestigio lingüístico a aquellas variedades que están más cercanas al poder económico y político.

Finalmente, es necesario señalar que este análisis pone de manifiesto la necesidad de gestar procesos de interculturalidad centrados en el reconocimiento de las diferencias culturales y la riqueza lingüística de los espacios rurales y urbanos del Cesar, del Caribe y de Colombia. De acuerdo con Islas (2005), los fenómenos lingüísticos merecen ser incluidos en cualquier estudio que tenga como fin prevenir la discriminación. En este sentido, este estudio podría considerarse un aporte para generar un escenario de formación para la paz, fundamentado en la aceptación del otro como un interlocutor válido en términos culturales, lingüísticos y sociales. La academia tiene el compromiso de fortalecer ese marco de formación para la paz como una propuesta política de reconocimiento, respeto de los derechos y la libertad de todos los seres humanos que ayude a erradicar la discriminación étnica, económica, social y, por supuesto, lingüística (Paz \& Díaz Pérez, 2019). 
Algunas actitudes lingüísticas hacia el español hablado en Valledupar (Colombia)

\section{Referencias bibliográficas}

1. Barrios, M. (9 de septiembre de 2019). Población de Valledupar creció 31 \%, revela DANE. El Heraldo. https:// www.elheraldo.co/cesar/poblacion-de-valledupar-crecio-31-revela-dane-664102

2. Bernal, J. (2016). Actitudes lingüísticas de los bogotanos hacia los dialectos del español hablado en Colombia y las variedades nacionales de esta lengua en los demás países de Hispanoamérica. University of Bergen. https:// www.bibliodigitalcaroycuervo.gov.co/1251/

3. Betancourt, A. \& García, C (1998). Caracterización fonética de dos zonas auríferas en Antioquia, Colombia. https://cvc.cervantes.es/lengua/ thesaurus/pdf/53/TH_53_001_013_0.pdf

4. Blanco, N. \& Pirela, J. (2015). La complementariedad metodológica: Estrategia de integración de enfoques en la investigación social. Espacios Públicos, 19(45). Universidad Autónoma del Estado de México. https://www. redalyc.org/pdf/676/67646966005.pdf

5. Blas Arroyo, J. (1999). Las actitudes hacia la variación intradialectal en la sociolingüística hispánica. Estudios Filológicos, 34, 47-72. https://scielo.conicyt.cl/scielo.php?script=sci_arttext\&pid=S0071-17131999003400005

6. Bryman, A. (2006). Integrating Quantitative and Qualitative Research: How It Is Done? London: Qualitative Research, Sage Publications.

7. Calderón, D. \& Gutiérrez, J. (2006). Caracterización sociolingüística de la comunidad de habla de Valledupar. Ítaca, 3(5), 156-171. Valledupar: Ediciones Unicesar.

8. Casanova, M. (2000). Análisis cualitativo y cuantitativo de la morfosintaxis de una segunda lengua: el caso del español en contacto con el catalán (Tesis doctoral). Lleida: Universidad de Lleida.

9. Campos, I. (2018). Lengua minorizada y enseñanza. Actitudes, metodologías y resultados de aprendizaje en el caso del aragonés. Zaragoza: Prensas de la Universidad de Zaragoza.

10. Castillo, M. (2006). El estudio de las actitudes de las actitudes en el contexto sociocultural: El caso del mexicano de Cuetzalan. Anales de Antropología, 40(1). http://www.revistas.unam.mx/index.php/antropologia/ article/view/9962/pdf_124

11. Céspedes, M. (2017). Estudio exploratorio en torno a la autopercepción del habla en contexto rural por parte de los habitantes rurales de la Provincia de Melipilla. https://www.redalyc.org/jatsRepo/5119/511954894001/ html/index.html

12. Cury Lambraño, J. (2004). La segunda muerte del verso. Departamento de Publicaciones de la Federación de Aseguradores Colombianos FASECOLDA.

13. Cury Lambraño, J. (2011). El Costeñol, un dialecto con toda la barba. Cartagena: Ediciones Pluma de Mompox S. A.

14. DANE (2018). Censo Nacional de Población y Vivienda 2018. Proyecciones de población 2018-2020. https:// www.dane.gov.co/index.php/estadisticas-por-tema/demografia-y-poblacion/proyecciones-de-poblacion 15. Drevdal, S. (2009). Actitudes lingüísticas de los inmigrantes nicaragüenses en Costa Rica hacia su propia habla 
Algunas actitudes lingüísticas hacia el español hablado en Valledupar (Colombia)

(Tesis de Licenciatura). Oslo: Universitetet i Bergen. http://bora.uib.no/bitstream/handle/1956/3822/56492314. pdf;jsessionid=E7A25FE5E098AC5D92A9CE71D0074497.bora-uib_worker?sequence=1

16. Edwards, J. (1982). Language Attitudes and Their Implications Among English Speakers. In Ryan, E. \& Giles, H. (Eds.), Attitudes towards Language Variation (pp. 20-33). London: Edward Arnold.

17. El Espectador (8 de marzo de 2000). El boom de las invasiones. https:/www.eltiempo.com/archivo/documento/ MAM-1297294

18. Erdösová, Z. (2011). El español de México en los ojos de sus hablantes: un estudio desde la sociolingüística y la dialectología perceptiva. Lengua y Voz, 1, 57-81 https://docplayer.es/12296420-El-espanol-de-mexico-en-losojos-de-sus-hablantes-un-estudio-desde-la-sociolinguistica-y-la-dialectologia-perceptiva.html

19. Estrada, R. \& Pérez, J. (2019). El gentilicio de Valledupar: actitudes lingüísticas hacia los términos vallenato y valduparense (Trabajo monográfico). Valledupar: Universidad Popular del Cesar.

20. Flórez, L. (1949). Cuestiones del español hablado en Montería y Sincelejo. Thesaurus, Tomo v, $124-162$. http://cvc.cervantes.es/lengua/thesaurus/indice.htm

21. Fundación Festival de la Leyenda Vallenata (2014). Reseña histórica de Valledupar. https://festivalvallenato. com/r-historica/

22. Gómez Molina, J. (2002). Lenguas en contacto y actitudes lingüísticas en la comunidad de Valencia. En J. Blas, M. Casanova, S. Fortuño \& M. Porcar (Eds.), Estudios sobre lengua y sociedad. Castellón de la Plana (España): Universitat Jaume I.http://books.google.com.co/books?id=PzDimeUxlOwC\&pg=PA57\&dq=componentes + de +1 $\mathrm{a}+$ actitud+linguistica\& $\mathrm{hl}=\mathrm{es} \& \mathrm{sa}=\mathrm{X} \& \mathrm{ei}=4 \mathrm{ubgT} 57 \mathrm{CA} 4 \mathrm{qu} 8 \mathrm{AS} 245 \mathrm{CHD}$ Q \& ved=0 C G Y Q 6 A EwCQ\#v=onepage\&q=componentes $\% 20 \mathrm{de} \% 201 \mathrm{a} \% 20$ actitud $\% 20$ linguistica $\& \mathrm{f}=$ false

23. González Cruz, I. (1995). Lengua, prestigio y prejuicios linguisticos: Algunas consideraciones sobre el espanol. Revue Belge de Philologie et d'Histoire, 73(3), 715-723. https://www.persee.fr/doc/rbph_0035-0818_1995_ num_73_3_4032

24. González, J. (2008). Metodología para el estudio de las actitudes. Actas del XXXVIIXXXVII Simposio Internacional de la Sociedad Española de Lingüistica (SEL). http://www.unav.es/linguis/simposiosel/actas 25. González, J. (2009). Actitudes lingüísticas en una comunidad rural: Els Ports (Castellón). Datos de un cuestionario sociolingüístico. Revista de Estudios Culturales, Vol. vIII, 75-95. http://www.erevistes.uji.es/index. $\mathrm{php} / \mathrm{clr} / \mathrm{article} / \mathrm{viewFile} / 64 / 62$

26. Hernández-Campoy, M. (s. f). El fenómeno de las actitudes y su medición en sociolingüistica. Universidad de Murcia. https://www.um.es/tonosdigital/znum8/portada/monotonos/04-JMCAMPOYa.pdf

27. Hernández-Campoy, J. M. \& Almeida, M. (2005). Metodología de la investigación sociolingüística. Málaga: Editorial Comares.

28. Islas, H. (2005). Lenguaje y discriminación. Cuadernos de la Igualdad. Consejo Nacional para Prevenir la Discriminación. https://www.conapred.org.mx/documentos_cedoc/CI004.pdf

29. Izquierdo, S. (2003). Actitudes ante el deterioro de la lengua. El español en Brasil. https://marcoele.com/ 
Algunas actitudes lingüísticas hacia el español hablado en Valledupar (Colombia)

descargas/13/izquierdo-deterioro_de_la_lengua.pdf

30. López Morales, H. (1989). Sociolingüística. Madrid: Gredos.

31. Makuc, M. (2011). La actitud lingüística en la comunidad de habla de Magallanes: aproximación a sus componentes básicos. Magallanía, 39(2), 105-111. https://scielo.conicyt.cl/scielo.php?script=sci_ arttext\&pid=S0718-22442011000200008

32. Martínez, S. (2017). El Festival de Leyenda Vallenata. Revista Credencial. http://www.revistacredencial.com/ credencial/historia/temas/el-festival-de-la-leyenda-vallenata

33. Montes, J. (1982). El español de Colombia. Propuesta de clasificación dialectal. Thesaurus. Tomo XXXVII. N. 1. http://cvc.cervantes.es/lengua/thesaurus/pdf/37/TH_37_001_023_0.pdf

34. Montes, J. (2012). El español de Colombia y el español de América. En Patiño, C. \& Bernal, J. (Coords.) El lenguaje en Colombia. Tomo 1: Realidad lingüística en Colombia, (pp. 3-10). Bogotá: Instituto Caro y Cuervo y Cuervo / Academia Colombiana de la Lengua.

35. Muñoz Navarrete, M. (s.f.). Supremacismo lingüístico.Rebelion.org.: http://www.rebelion.org/docs/87719. pdf

36. Ocampo, G. (2003). Urbanización por invasión. conflicto urbano, clientelismo y resistencia en Córdoba (Colombia). Revista Colombiana de Antropología, 39, 237-272. http://www.scielo.org.co/scielo.php?script=sci_ arttext\&pid=S0486-65252003000100008

37. Olmos, A. Gómez, T \& Pérez A. (2010). La variación sociolingüística de /s/ implosiva en la comunidad de habla de Valledupar, Colombia. Valledupar: Universidad Popular del Cesar. https://www.ellibrototal.com/ ltotal $/ ? \mathrm{t}=1 \& \mathrm{~d}=5392$

38. Paz, E \& Díaz Pérez, W. (2019). Educación para la paz: una mirada desde la Universidad Nacional Autónoma de Honduras. Innovación Educativa, 19(79), 171-195. http://www.scielo.org.mx/scielo.php?script=sci_ arttext\&pid=S1665-26732019000100171\&lng=es\&tlng=es.

39. PRESEEA (2003). Metodología general. http://preseea.linguas.net/Portals/0/Metodologia/ METODOLOG\%C3\%8DA\%20PRESEEA.pdf

40. Real Academia Española (2020). Diccionario de la lengua española. https://dle.rae.es/montuno

41. Rodríguez, Y. (1999). Marcadores discursivos en el habla de Barranquilla. Litterae. Revista de la Asociación de Exalumnos del Seminario Andrés Bello del ICC, 8, 197-220.

42. Rojo, G. (1979). Aproximación a las actitudes lingüísticas del profesorado de E.G.B. en Galicia, Instituto de Ciencias de la Educación de la Universidad de Santiago. https:/gramatica.usc.es/ grojo/Publicaciones/ Aproximacion_actitudes_linguisticas_profesorado_EGB.pdf

43. Salazar, A. (2019). Solamente porque uno habla goppiao lo tratan de corroncho: creencias de los monterianos acerca del español hablado en Montería. Revista de Lingüística y Literatura, 76. Universidad de Antioquia. https:// aprendeenlinea.udea.edu.co/revistas/index.php/lyl/article/view/340077/20794857

44. SalgadoLévano,A.(2007).Investigacióncualitativa:diseños, evaluacióndelrigormetodológicoyretos.Liberabit, 
Algunas actitudes lingüísticas hacia el español hablado en Valledupar (Colombia)

13(13), 71-78. http://www.scielo.org.pe/scielo.php?script=sci_arttext\&pid=S1729-48272007000100009

45. Serrano, I. (2017). Los de Guadalajara hablamos «cantadito». Iván Bien. http://ivanbien.com/2017/02/13/losde-guadalajara-hablamos-cantadito/

46. Silva-Corvalán, C \& Arias, A. (2017). Sociolingüistica y pragmática del español. Washington D.C.: Georgetown University Press.

47. Terraza, L. (2020). Caracterización de la consonante vibrante simple alveolar sonora / $r$ / en Valledupar (Colombia). (Trabajo monográfico). Valledupar: Universidad Popular del Cesar.

48. Trillos, M. (2012). El español allende la mar de los caribes. El Lenguaje En Colombia. Tomo I. Bogotá: Imprenta Patriótica del Instituto Caro y Cuervo.

49. Trudgill, P. (1983) Sociolinguistics: An Introduction to Language and Society. London: Penguin. 50. Valledupar.com. (s.f.). Datos de nuestra ciudad. https://www.valledupar.com/laciudad.html 